\title{
Kikuchi-Fujimoto disease mimicking tuberculous lymphadenitis or lymphoma on fluorine-18 fluorodeoxyglucose positron emission tomography/computed tomography
}

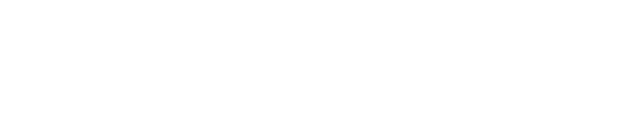

\author{
Noraini Abdul Rahim' \\ Abdul Jalil Nordin ${ }^{2}$ \\ Chiara Rusconi ${ }^{3}$ \\ Cristina Gabutti ${ }^{3}$ \\ Erika Ravelli ${ }^{4}$ \\ Claudio Rossetti ${ }^{5}$ \\ 'Diagnostic Imaging Department, \\ Serdang Hospital, Selangor, \\ ${ }^{2}$ Centre for Diagnostic Nuclear \\ Imaging, University Putra Malaysia, \\ Serdang, Malaysia; ${ }^{3}$ Department of \\ Haematology, Ospedale Niguarda, \\ Milan, ${ }^{4}$ Unit of Haematology, Valduce \\ Hospital, Como, ${ }^{5}$ Department of \\ Advance Technology (Nuclear \\ Medicine), Ospedale Niguarda, Milan, \\ Italy
}

\begin{abstract}
Kikuchi-Fujimoto disease, also known as histiocytic lymphadenitis is a rare and benign disease characterized by regional cervical lymphadenopathy with fever of unknown origin, affecting mainly young women. We describe a case of a young Asian female who was initially misdiagnosed as having tuberculous lymphadenitis because of multiple lymphadenopathies with 18-fluorodeoxyglucose uptake on positron emission tomography/computed tomography. Final histologic diagnosis of Kikuchi-Fujimoto disease was made after excisional biopsy of the affected lymph node. The cervical lymphadenopathy has reduced in size and lung lesions disappeared after 2 months without specific treatment.
\end{abstract}

Keywords: cervical lymphadenopathy, Kikuchi disease, PET/CT features

\section{Case report}

A 22-year-old female of Taiwanese descent presented to the Ospedale Niguarda Ca' Granda, Milan, Italy with complaints of pain in the throat, associated fever, and sweats of 2 months' duration. No significant past medical and surgical history was obtained. There was also no history of recent travels. Ten days later, she developed a $2 \mathrm{~cm}$ swelling in the right cervical region. Ultrasound of the neck showed multiple oval hypoechoic lesions with ill-defined margins seen in the right lateral cervical and parotid region. Ultrasound of the abdomen revealed no abnormality. The physical examination of other systems was normal. Subsequently, 1 month later, the patient presented again with fever, physical examination then revealed fullness of the nasopharynx and persistent lateral cervical lymphadenopathy. Patient was febrile. At this point, positron emission tomography/computed tomography (PET/CT) using fluorine-18 fluorodeoxyglucose (18F-FDG) tracer was performed and showed conglomerate FDG avid lymphadenopathies in the right cervical region and a well-defined solitary nodule in the upper lobe of the right lung with high FDG activity. Blood investigations revealed white blood count of $9,000 / \mu \mathrm{L}$, neutrophil of $14.2 \mathrm{~g} / \mathrm{dL}$, platelet count of $404 \times 10^{9} / \mathrm{L} \mu \mathrm{L}$ with normal liver and renal function tests. She was attended by the hematologist and received a course of oral antibiotics (Augmentin) which was changed to intramuscular Bactrim and Rocephin due to persistent temperature. Written informed consent was obtained from the patient before conducting the study. Ethical approval from the Ethics Committee of Ospedale Niguarda Ca' Granda was not required for writing this case report.
Correspondence: Abdul Jalil Nordin Centre for Diagnostic Nuclear Imaging, Universititi Putra Malaysia, 43400 Serdang, Malaysia Email drimaging@yahoo.com
Dovepress

http://dx.doi.org/10.2147/COAYA.S69923 (c) (i) (5) 2016 Abdul Rahim et al. This work is published and licensed by Dove Medical Press Limited. The full terms of this license are available at https://www.dovepress.com/

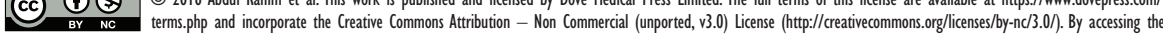
work you hereby accept the Terms. Non-commercial uses of the work are permitted without any further permission from Dove Medical Press Limited, provided the work is properly attributed. For permission for commercial use of this work, please see paragraphs 4.2 and 5 of our Terms (https://www.dovepress.com/terms.php). 


\section{A}

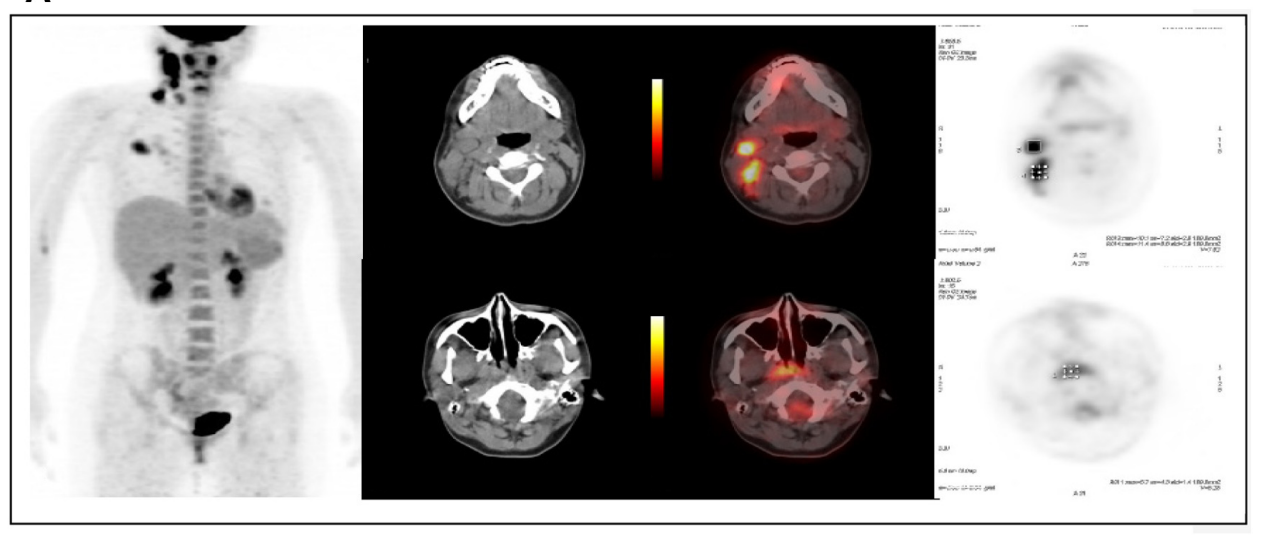

B

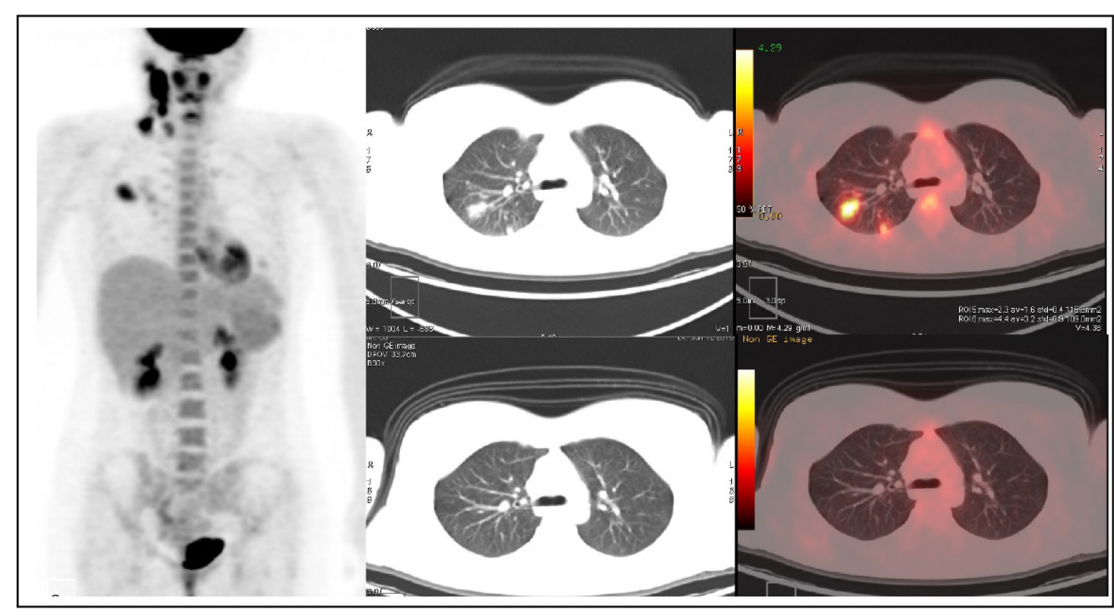

Figure I Fluorine-18 fluorodeoxyglucose PET/CT MIP and cross sectional images at the head and neck region and transthoracic level.

Notes: (A) The first PET/CT was carried out to rule out lymphoproliferative disease, it showed multiple small lesions in right lateral cervical and supraclavicular regions. It showed high 18-fluorodeoxyglucose uptake on the right side of the nasopharynx and a biopsy confirmed lymphoid hyperplasia of the palatine tonsil. (B) Two lesions in the right lung field. They are likely to represent an infectious foci.

Abbreviation: PET/CT, positron emission tomography/computed tomography.

The serum tests for toxoplasmosis and Epstein-Barr virus were negative. The serological titers for cytomegalovirus were positive. Purified protein derivative was positive. Serum immuno-stain markers correlated well with lymphoid hyperplasia. Biopsy of the roof of the nasopharyngeal lesion was done and histology was found compatible with lymphoid hyperplasia of the palatine tonsil.
Suspecting a lymphoproliferative disease, another 18F-FDG PET/CT was carried out which showed multiple small lesions in right lateral cervical and supraclavicular regions and two in the right pulmonary fields. A repeat ultrasound of the neck after 1 month showed multiple right lateral cervical nodes of increased dimensions compared to the previous examination. The underlying pathology was

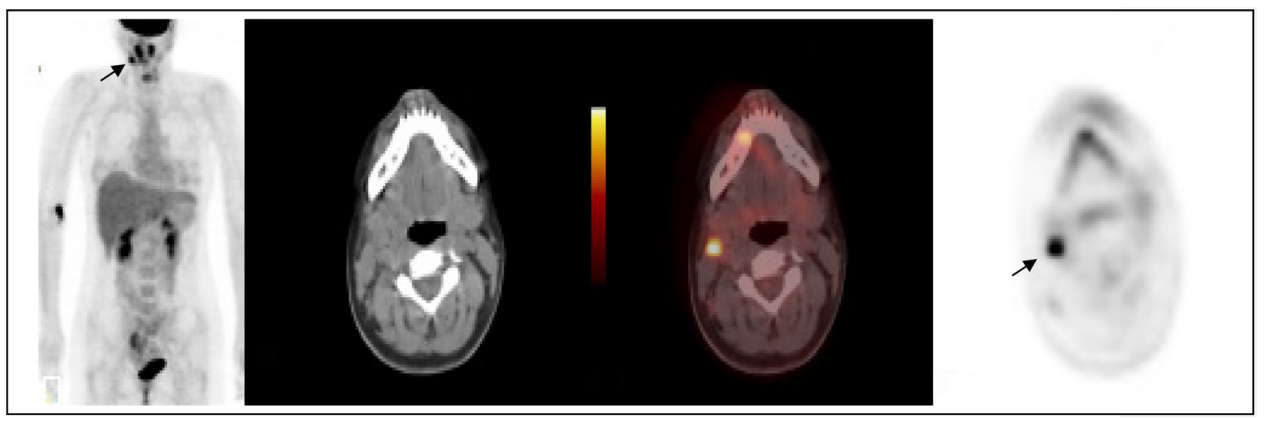

Figure 2 I8F-FDG PET/CT at 2 month intervals show reduction in size and number of cervical nodes (arrow) and that lesions in the lung field had disappeared. Abbreviation: PET/CT, positron emission tomography/computed tomography. 
still uncertain. An excisional biopsy of the cervical node was done and histopathology examination reported past inversion of cellular architecture with immunochemistry positive to myeloperoxidase and partially to $\mathrm{CD} 68 \mathrm{r}$, tissue proliferative rate evaluation with MIB1 findings were compatible with Kikuchi-Fujimoto disease (KFD) with a high number of blast cells. Bone marrow aspiration and biopsy did not reveal any abnormality. Other causes of lymphadenopathies were also considered such as tuberculosis infection or other viral infections. Peripheral blood serology turned out positive for human herpes simplex virus IgM antibodies. Peripheral blood smear and autoimmunity tests were normal.

Considering the strong possibility of KFD, the patient did not receive any specific treatment. A repeat PET/CT performed after 2 months showed reduction in size and number of the cervical focal uptakes, and was negative in other areas including the right lung field. She remained well on review at the clinic 5 months later, and latest PET/CT after 9 months was found to be negative.

\section{Discussion}

$\mathrm{KFD}$, also known as necrotizing histiocytic lymphadenitis, is a rare disease first described in 1972 by Fujimoto et $\mathrm{al}^{1}$ and Kikuchi. ${ }^{2}$ This disorder typically affects young women and shows a high prevalence among Asian people. It is a benign and self-limited disease, presenting with regional lymphadenopathies usually accompanied by constitutional symptoms like fever, frequently associated with upper respiratory tract symptoms. Less common symptoms are night sweats, malaise, fatigue, nausea, vomiting, and weight loss. The onset can be acute or sub-acute, usually developing during a period of 2-3 weeks, and at times lasting for 6-8 weeks. Etiology remains unclear. Many cases have reported associations with autoimmune disorders. Systemic lupus erythematosus is often associated with it, thus in KFD patients close monitoring and follow-up to investigate for autoimmune diseases has been recommended. Some viruses are involved, in particular cytomegalovirus, Epstein-Barr virus, herpes simplex virus, and herpes zoster virus.

Because of the nature of the disease, it is challenging to diagnose KFD as it is frequently mistaken for malignant lymphoma. Final diagnosis is made by histological analysis of lymph node biopsy and no pathognomonic laboratory tests are available. It is characterized histopathologically by cortical and paracortical necrosis with lymphoreticular infiltration and absence of granulocytic infiltration.

There are no specific clinical or radiological features of KFD. The CT features of homogenously enhancing nodes without significant nodal necrosis make radiological differentiation from tuberculosis, metastases, or lymphoma impossible.

Lung involvement in KFD is unusual. Garcia-Zamalloa et $\mathrm{al}^{3}$ reported a case of pleural effusion and interstitial fibrosis complicating KFD. Fatal lung involvement has also been reported previously with cryptogenic organizing pneumonia and pulmonary hemorrhage..$^{-7}$ Migratory type of KFD was reported by Al Attia et $\mathrm{al}^{8}$ involving cervical, occipital, and jugulodigastric nodes. Recurrent KFD was sporadically mentioned in most case reports, but the actual rate is not known. It is also not known if KFD can transform into malignancy. In recent years, usefulness and usage of 18F-FDG PET in KFD has been reported. It has been shown that in KFD, lymph nodes have an uptake of FDG similar to aggressive lymphoma ${ }^{9-11}$ and other chronic granulomatous diseases like tuberculous lymphadenitis. This study can help clinicians to identify the severity of the disease through extension of lymph node involvement. Biopsy is mandatory, which is useful in assessing patient progress following treatment and confirming 18F-FDG PET/CT findings, especially during follow-up. The fact that KFD lesions demonstrate high 18F-FDG uptake on PET/CT, means that this can potentially be a future modality of choice for monitoring treatment response in KFD patients.

\section{Conclusion}

On diagnostic imaging including 18F-FDG PET/CT of the cervical lymphadenopathy in KFD, the findings can mimic lymphoma, metastases, or tuberculosis. In relatively young patients especially of Asian origin, KFD is a possible differential diagnosis and biopsy should be done to confirm the diagnosis.

\section{Disclosure}

The authors report no conflicts of interest in this work.

\section{References}

1. Fujimoto Y, Kozima Y, Yagamuchi K. Cervical subacute lymphadenitis: a new clinicopathologic entity. Naika. 1972;30:920-927.

2. Kikuchi M. Lymphadenitis showing focal reticular cell hyperplasia with nuclear debris and phagocytosis. Acta Hematol Jpn. 1972;35:379-380.

3. Garcia-Zamalloa A, Taboada-Gomez J, Bernardo-Galán P, Magdalena FM, Zaldumbide-Dueñas L, Ugarte-Maiztegui M. Bilateral pleural effusion and interstitial lung disease as unusual manifestations of KikuchiFujimoto disease: case report and literature review. BMC Pulm Med. 2010;10:54

4. Sharma OP. Unusual systemic disorders associated with interstitial lung disease.Curr Opin Pulm Med. 2001;7(5):291-294.

5. Chen HC, Lai JH, Huang GS, et al. Systemic lupus erythematosus with simultaneous onset of Kikuchi-Fujimoto's disease complicated with antiphospholipid antibody syndrome: a case report and review of the literature. Rheumatol Int. 2005;25(4):303-306. 
6. Kucukardali Y, Solmazgul E, Kunter E, Oncul O, Yildirim S, Kaplan M. Kikuchi-Fujimoto disease: analysis of 244 cases. Clin Rheumatol. 2007;26(1):50-54.

7. Hua F, Zhu L. Kikuchi-Fujimoto disease associated with cryptogenic organizing pneumonia: case report and literature review. BMC Infect Dis. 2010;10:64.

8. Al Attia HM, Al Ahmed YH, El Hag A, El Abassi RN. A case of migratory lymphadenopathy and cutaneous anergy in an Asian woman. Postgrad Med J. 2000;76(900):660-663.
9. Kaicker S, Gerard PS, Kalburgi S, Geller MD, Hailoo D. PET-CT scan in a patient with Kikuchi disease. Pediatr Radiol. 2008;38(5):596-597.

10. Ito K, Morooka M, Kubota K. F-18 FDG PET/CT findings showing lymph node uptake in patients with Kikuchi disease. Clin Nucl Med.2009;34(11): $821-822$.

11. Kim CH, Hyun OJ, Yoo IeR, Kim SH, Sohn HS, Chung SK. Kikuchi Disease Mimicking Malignant Lymphoma on FDG PET/CT. Clin Nucl Med. 2007;32(9):711-712.
Clinical Oncology in Adolescents and Young Adults

\section{Publish your work in this journal}

Clinical Oncology in Adolescents and Young Adults is an international, peer-reviewed, open access journal publishing original research, reports, editorials, reviews and commentaries on all aspects of epidemiology, diagnosis and treatment of cancers in adolescents and young adults. The manuscript management system is completely

\section{Dovepress}

online and includes a very quick and fair peer-review system. Visit http://www.dovepress.com/testimonials.php to read real quotes from published authors. 